\title{
Acknowledgement to Reviewers of Biomedicines in 2016
}

Biomedicines Editorial Office

MDPI AG, St. Alban-Anlage 66, 4052 Basel, Switzerland; biomedicines@mdpi.com

Published: 12 January 2017

The editors of Biomedicines would like to express their sincere gratitude to the following reviewers for assessing manuscripts in 2016.

We greatly appreciate the contribution of expert reviewers, which is crucial to the journal's editorial process. We aim to recognize reviewer contributions through several mechanisms, of which the annual publication of reviewer names is one. Reviewers receive a voucher entitling them to a discount on their next MDPI publication and can download a certificate of recognition directly from our submission system. Additionally, reviewers can sign up to the service Publons (https://publons.com) to receive recognition. Of course, in these initiatives we are careful not to compromise reviewer confidentiality. Many reviewers see their work as a voluntary and often unseen part of their role as researchers. We are grateful to the time reviewers donate to our journals and the contribution they make.

If you are interested in becoming a reviewer for Biomedicines, see the link at the bottom of the webpage http://www.mdpi.com/reviewers.

The following reviewed for Biomedicines in 2016:

$\begin{array}{lll}\text { Abba, Katharine } & \text { Dingermann, Theodor } & \text { Lundstrom, Kenneth } \\ \text { Alemany, Ramon } & \text { Donnelly, Oliver } & \text { MacNeill, Amy } \\ \text { Andrianasolo, Eric } & \text { Ganesan, A. } & \text { Mcfadyen, Morag } \\ \text { Antignani, Antonella } & \text { García-Martín, María L. } & \text { Michel, Martin C. } \\ \text { Arens, Ramon } & \text { Girbés, Tomás } & \text { Mirsayar, MirMilad } \\ \text { Atkins, Gregory J. } & \text { González, Víctor M. } & \text { Nakagawa, Yoshimi } \\ \text { Barbieri, Federica } & \text { Gunnarsson, Anders } & \text { Nelson, Delia J. } \\ \text { Baum, Richard P. } & \text { Hanson, Robert N. } & \text { Nuño, Natalia } \\ \text { Berkhout, Ben } & \text { Hingorani, Dina } & \text { Phyo, Aung Pyae } \\ \text { Borlak, Jürgen } & \text { Hirata, Kenji } & \text { Puddu, Paolo Emilio } \\ \text { Busardo, Francesco } & \text { Hu, Jinming } & \text { Quintarelli, Concetta } \\ \text { Call, Mindy } & \text { Jayant, Rahul Dev } & \text { Rabkin, Samuel David } \\ \text { Capasso, Raffaele } & \text { Jordan, V. Craig } & \text { Ramírez, Manuel } \\ \text { Casco-Robles, Martin M. } & \text { Kasoju, Naresh } & \text { Rein, Alan } \\ \text { Cerullo, Vincenzo } & \text { Kobe, Carsten } & \text { Rovero, Paolo } \\ \text { Chuburu, Françoise } & \text { Kuehnel, Florian } & \text { Samal, Siba } \\ \text { Chudasama, Vijay } & \text { Kunjachan, Sijumon } & \text { Schwartz, Jean-Louis } \\ \text { Cornetta, Kenneth } & \text { LaFace, Drake } & \text { Serda, Rita Elena } \\ \text { Costantino, Luca } & \text { Layer, Paul G. } & \text { Seymour, Len W. } \\ \text { Crickmore, Neil } & \text { Leporatti, Stefano } & \text { Sherker, Averell H. } \\ \text { Cripe, Timothy P. } & \text { Leung, Chung-Hang } & \text { Sikora, Andrew } \\ \text { Damour, Odile } & \text { Li, Qingliang } & \text { Smerdou, Cristian } \\ \text { Das, Soumen } & \text { Löchelt, Martin } & \text { Sousa, Célia T. } \\ \text { Davila, Marco L. } & \text { Lorenzi, Vannina } & \text { Tai, Dar-Fu } \\ \text { Delville, Marie-Hélène } & \text { Loskog, Angelica } & \text { Taïeb, David } \\ & & \end{array}$


Tanner, Julian A.

Tatullo, Marco

Vähä-Koskela, Markus

Valable, Samuel

Van Den Wollenberg, Diana J. M.

\author{
Vankemmelbeke, Mireille \\ Vassilopoulos, George \\ Villalona-Calero, Miguel \\ Wan, Yonghong \\ $\mathrm{Wu}$, Lianfeng
}

\author{
Wujcik, Evan \\ Yamada, Akira \\ Yan, Guangmei \\ Zhou, Heshan Sam
}

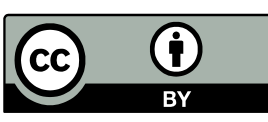

(C) 2017 by the authors; licensee MDPI, Basel, Switzerland. This article is an open access article distributed under the terms and conditions of the Creative Commons Attribution (CC-BY) license (http://creativecommons.org/licenses/by/4.0/). 